\title{
A relaÇão entre álcool, tabaco e eSTResSe em eSTUdantes de ENFERMAGEM
}

\author{
Marcos Hirata Soares ${ }^{1}$ \\ Felipe Santana Oliveira ${ }^{2}$
}

Objetivo: este estudo caracteriza o estresse do estudante de enfermagem, sua relação com o processo de formação acadêmica e com o uso de substâncias psicoativas. Métodos: trata-se de estudo descritivo, transversal, com 149 estudantes, em Londrina, PR, em 2012. Foram utilizados a escala Avaliação de Estresse no Estudante de Enfermagem e o Teste de Triagem do Envolvimento com Álcool, Tabaco e outras Substâncias Psicoativas. A análise foi feita com o Statistical Package for Social Sciences, versão 15, pelos testes de Kruskal-Wallis, Jonckheere-Terpstra e correlação de Spearman, em nível $\alpha<0,05$. Resultados: identificou-se maior prevalência no consumo do tabaco, diferenças entre o nível de estresse no decorrer das séries, uma relação negativa fraca entre o estresse e o consumo de tabaco e uma relação positiva fraca quanto ao consumo de álcool e estresse. Conclusão: o estudo contribuiu para descrever as relações entre o estresse, o processo de formação acadêmica e o uso de álcool e tabaco.

Descritores: Estresse Psicológico; Estudantes de Enfermagem; Instituições de Ensino Superior; Detecção do Abuso de Substâncias.

\footnotetext{
1 MSc, Professor, Departamento de Enfermagem, Centro de Ciências da Saúde, Universidade Estadual de Londrina, Londrina, PR, Brasil.

${ }^{2}$ Aluno do curso de graduação em Enfermagem, Departamento de Enfermagem, Centro de Ciências da Saúde, Universidade Estadual de Londrina, Londrina, PR, Brasil.
} 


\section{THE RELATION BETWEEN ALCOHOL, TOBACCO AND STRESS IN NURSING STUDENTS}

Objective: This study characterizes stress of the Nursing student, its relation with the academic learning process and the use of psychoactive substances. Methods: This is a transversal, descriptive study with 149 students, in Londrina-PR, in 2012. We used the Stress Scale Evaluation on the Nursing Student and the Alcohol, Smoking and Substance Involvement Screening Test. The analysis was done with the Statistical Package for Social Sciences, v.15, through Kruskal-Wallis, Jonckheere-Terpstra tests and Spearman Correlation, in level $\alpha<0.05$. Results: We identified a higher prevalence of tobacco consumption, differences in the level of stress during the series, a weak negative correlation between stress and tobacco, and a weak positive correlation regarding the consumption of alcohol and stress. Conclusion: The study has contributed to describe the relations between stress, the academic learning process and the use of alcohol and tobacco.

Descriptors: Stress, Psychological; Nursing, Students; Higher Education Institutions; Substance Abuse Detection.

\section{LA RELACIÓN ENTRE ALCOHOL, TABACO Y ESTRÉS EN ESTUDIANTES DE ENFERMERÍA}

Objetivo: este estudio caracteriza el estrés del estudiante de Enfermería, su relación con el proceso de formación académica y con el uso de substancias psicoactivas. Métodos: se trata de un estudio descriptivo, transversal con 149 estudiantes, en Londrina-PR, en 2012. Fueron utilizados la escala Evaluación de Estrés en el Estudiante de Enfermería y la Prueba de Tría del Envolvimiento con Alcohol, Tabaco y otras Substancias Psicoactivas. El análisis fue hecho con el Statistical Package for Social Sciences, v.15, por los testes de Kruskal-Wallis, Jonckheere-Terpstra y Correlación de Spearman, en nivel a $<0.05$. Resultados: se identificó mayor superioridad en el consumo del tabaco, diferencias entre el nivel de estrés en el pasar de las series, una relación negativa flaca entre el estrés y el consumo de tabaco y una relación positiva flaca en cuanto al consumo de alcohol y estrés. Conclusión: el estudio aportó para describir las relaciones entre el estrés, el proceso de formación académica y el uso de alcohol y tabaco.

Descriptores: Estrés Psicológico; Estudiantes de Enfermería, Instituciones de Enseñanza Superior; Detección de Abuso de Substancias.

\section{Introdução}

De acordo com alguns estudos sobre o diagnóstico de abuso ou dependência de sustâncias psicoativas, como o álcool e o tabaco em estudantes universitários ${ }^{(1-2)}, \mathrm{o}$ contexto universitário, particularmente para o estudante de enfermagem, torna-o mais vulnerável a vivenciar o abuso de substâncias ilícitas ${ }^{(3-4)}$. Dentre os mais diversos fatores relacionados ao abuso de substâncias psicoativas, como os fatores psicológicos, por exemplo, o enfrentamento 
do estresse pode também ser considerado como fator associado.

\section{Revisão de literatura}

Em dois estudos sobre estresse em estudantes de enfermagem $^{(5-6)}$, identificaram-se que as maiores fontes de estresse para o acadêmico são relacionadas aos estudos e atividades acadêmicas e preocupação com sua inserção no mercado de trabalho, bem como sobrecarga no último ano do curso $^{(6)} \mathrm{e}$, consonante a esse fato, uma pesquisa ${ }^{(4)}$ identificou que o consumo de tabaco aumentava gradativamente nas séries, assim como práticas de automedicação, sugerindo a hipótese de que a complexidade acadêmica configuravase como fonte estressora, fazendo com que os estudantes utilizassem, como forma de enfrentamento, as substâncias lícitas e ilícitas.

Refletindo sobre o tema estresse, considera-se que teorias de enfrentamento do estresse são importantes para servirem como uma das ferramentas de compreensão e investigação para os enfermeiros. Há dois grandes principais modelos de abordagem para o estresse, sendo um com ênfase no modelo de respostas fisiológicas, classificando-as em alarme, resistência e exaustão ${ }^{(7)}$, e o outro apoia-se em um modelo de adaptação e enfrentamento do estresse ${ }^{(8)}$, o qual focaliza as respostas psicológicas. Tal modelo foi escolhido por ser considerado mais adequado a este estudo, pois define que o enfrentamento ao estresse, considerado um processo, é condicionado às reavaliações contínuas que o indivíduo faz de seu relacionamento com o meio, sendo esse envolvido pelas características da personalidade, valores, crenças, fatores socioculturais e eventos de vida.

Nessa relação complexa de enfrentamento, há a possibilidade de envolvimento do sujeito com substâncias psicoativas, as quais podem ser utilizadas como formas de enfrentamento ao estresse ou outro sofrimento psicológico pelo qual o estudante esteja vivencindo.

Quando o enfrentamento ao estresse é bem-sucedido, é possível mudança real de relacionamento entre o sujeito e seu meio, permitindo a adaptação, a qual afeta as áreas de saúde, bem-estar psicológico e funcionamento social de forma positiva. No entanto, a dinâmica do curso de graduação em enfermagem, com seus variados momentos de complexidade teórica e prática, tem como uma das suas características, ser um curso com momentos variados de intensidade e fontes de estresse. Nesse aspecto, uma pesquisadora desenvolveu um instrumento de identificação e mensuração do estresse acadêmico do estudante de enfermagem $^{(9)}$, estratificando-os de baixo a muito alto nível de estresse e as respectivas áreas que são percebidas como estressoras.

Considerando a relevância e magnitude do estresse que, segundo um levantamento ${ }^{(10)}$, sugere ser maior na enfermagem, quando comparada a outras áreas, é de grande importância caracterizar o tipo e a prevalência de estresse entre as séries do curso de graduação em enfermagem, bem como sua relação com o abuso de substâncias psicoativas. Neste estudo, considerou-se importante verificar a associação entre estresse acadêmico e o uso de álcool e tabaco, utilizando o instrumento Avaliação do Estresse do Estudante de Enfermagem (AEEE) ${ }^{(9)}$, e do Teste de Triagem do Envolvimento com Álcool, Tabaco e outras Substâncias (Assist), um teste que mensura a frequência do uso de substâncias psicoativas e a necessidade de intervenção ${ }^{(11)}$. Dessa forma, o presente estudo teve como objetivo verificar a associação entre o nível de estresse do graduando de enfermagem, o consumo de álcool e tabaco e entre o nível de estresse e a série do curso.

\section{Métodos}

Trata-se de estudo descritivo, transversal e individuado. A amostra foi composta por 149 alunos, distribuídos em todas as séries do curso de graduação em enfermagem de uma instituição de ensino superior, no interior do Paraná, no período de junho a agosto de 2012. Os momentos de coleta de dados foram adaptados à dinâmica e rotina de cada série, convidando-os, individualmente, nos horários vagos de atividades acadêmicas.

Os critérios de inclusão foram ser aluno regularmente matriculado no curso. O projeto foi aprovado pelo Comitê de Ética em Pesquisa com Seres Humanos da Universidade Estadual de Londrina (C.A.A.E. 028.0.268.000-11).

OtesteAssisté um teste validado internacionalmente ${ }^{(12)}$ e no Brasil ${ }^{(11)}$, com alfa de Cronbach de 0,80. Possui o objetivo de avaliar o envolvimento com drogas lícitas e ilícitas, direcionando a necessidade de tratamento pela intervenção breve ou encaminhamento no caso de provável dependência química. No entanto, o presente estudo não teve o objetivo da aplicação do instrumento para aplicação da intervenção, por não se tratar do objetivo da pesquisa, mas, a partir do momento que houve conhecimento de estudantes com problemas relacionados ao álcool e tabaco, os mesmos foram encaminhados aos serviços disponíveis na universidade.

O instrumento de mensuração dos níveis de estresse, denominado instrumento para Avaliação de Estresse em Estudantes de Enfermagem AEEE) ${ }^{(9)}$, propõe que o estresse está vinculado à relação entre indivíduo e seu ambiente e o seu estudo requer que as pessoas sejam avaliadas dentro de seus próprios contextos, em sua relação com o ambiente e na atribuição de significados aos eventos. O AEEE, cuja consistência interna estimada pelo alfa de Cronbach variou de 0,71 a 0,87, permite avaliar o estresse entre estudantes de enfermagem como variável em estudos ou para finalidades educacionais por meio de seis domínios.

O domínio 1 (D1), Realização de Atividades Práticas, refere-se ao conhecimento instrumental adquirido pelo aluno para a realização dos procedimentos e os sentimentos envolvidos no momento da assistência ao paciente. No domínio 2 (D2), Comunicação Profissional, são avaliadas as dificuldades sentidas na comunicação dentro do local de trabalho e nas situações de conflito que surgem. O domínio 3 (D3), Gerenciamento do Tempo, considera a dificuldade do aluno em conciliar as atividades acadêmicas estabelecidas na grade curricular com as exigências pessoais, emocionais e sociais. No domínio 4 (D4), Ambiente, aborda o grau de dificuldade sentido no acesso aos campos de estágio ou 
universidade e às situações de desgaste percebidas pelos alunos com os meios de transporte utilizados. Os itens do domínio 5 (D5), Formação Profissional, referem-se à preocupação do aluno sobre o conhecimento adquirido em sua fase de formação acadêmica e o impacto que esse exerce sobre sua futura vida profissional, além de abordar também sua percepção em situações que poderá viver quando profissional. Já no domínio 6 (D6), Atividade Teórica, mede o grau de dificuldade do aluno ao lidar com o conteúdo programático, as atividades desenvolvidas e a metodologia de ensino adotada.

Os dados foram analisados pelo programa Statistical Package for Social Sciences (SPSS), versão 21. O passo inicial foi realizar um teste de normalidade, visando escolher entre testes paramétricos, para distribuição normal, e não paramétricos, para não normal, antes de se iniciar a análise propriamente dita. Os testes aplicados para avaliar a normalidade da amostra foram os testes de Kolmogorov-Smirnov e Shapiro-Wilk, com correção de Lilliefors, cujos resultados variaram entre $p=0,000$ e $\mathrm{p}=0,314$, classificando a amostra como não normal, de forma global.

A comparação entre as séries foi realizada pelo teste de Kruskal-Wallis, que é indicado para comparações entre três ou mais grupos. A busca por uma relação linear de tendência positiva ou negativa entre o avançar das séries e o nível de estresse foi verificada pelo teste de JonckheereTerpstra e, a fim de conhecer as possíveis correlações entre as variáveis, utilizou-se o coeficiente de correlação de Spearman. O nível de significância adotado foi $\alpha<0,05$, sendo que também foi admitido o nível $\alpha<0,01$, na correlação de Spearman.

A correlação de Spearman busca a existência de uma relação linear entre duas variáveis ordinais, podendo expressar se há uma relação positiva, ou seja, quanto mais uma variável aumenta, mais a outra variável relacionada também aumenta, ou pode expressar também uma relação negativa, significando que quanto mais uma variável aumenta mais a outra variável diminui. Dessa forma, a correlação expressa a intensidade da relação entre duas variáveis e quanto maior o coeficiente de correlação, maior a intensidade dessa relação, cujo valor varia entre -1 e +1. A interpretação de valores varia de acordo com cada autor. Neste estudo, valores de 0 a 0,20 indicam uma correlação muito baixa, de 0,20 a 0,40 , baixa, entre 0,40 e 0,60 indicam correlação moderada, entre 0,60 e 0,80 , alta, e 0,80 a 1,0, muito alta $^{(13)}$.

\section{Resultados}

\section{Álcool e Tabaco}

Foram entrevistados 149 alunos da graduação de enfermagem de uma universidade pública do Paraná, equivalendo a $67 \%$ do total de estudantes, sendo a idade mínima de 18 anos e a máxima de 39, $(\mathrm{m}=21,5 ; \mathrm{dp} \pm 3$ anos). Nessa amostra, 92 (61\%) moravam longe da família em repúblicas ou pensões, enquanto 57 (39\%) viviam com a família. Houve participação homogênea de todos os anos da graduação: o primeiro ano com $28 \%$, o segundo ano com $22 \%$, o terceiro ano com $26 \%$ e o quarto ano com $24 \%$ de cada série.

Os dados mensurados pelo Assist (Tabela 1) foram representados, no caso do álcool, com 0 a 3 pontos, classificados como baixo risco, 4 a 26 pontos, médio risco e acima de 26 pontos, alto risco ou provável dependência do álcool. Para o tabaco, 0 a 10 pontos representam baixo risco, 11 a 26, médio risco, e acima de 26, alto risco ou provável dependência do tabaco. Para a escala AEEE, quanto ao escore total, a pontuação mínima foi de 27 , a máxima de $78(\mathrm{~m}=51,93 ; \mathrm{dp}=10,03)$.

A comparação entre as séries, quanto aos domínios de estresse, resultou nos valores de $X^{2}=12,752, \mathrm{p}=0,005$, para o domínio D5 e 11,622, p=0,009, para o domínio D6 no teste de Kruskal-Wallis. Quanto ao teste de Jonckheere-Terpstra, foram os valores de 3,041, $\mathrm{p}=0,002$, para o domínio D5 e $-3,321, \mathrm{p}=0,001$, para o domínio D6. Foram apresentados apenas os resultados estatisticamente significativos, por serem considerados mais importantes.

Tabela 1 - Porcentagem e frequência do consumo referido de álcool e tabaco por graduandos de enfermagem. Londrina, PR, Brasil, 2012

\begin{tabular}{|c|c|c|c|c|c|}
\hline & Baixo risco & Médio risco & Alto risco & Média & Desvio-padrão \\
\hline Álcool & $41(27,5 \%)$ & $103(69,1 \%)$ & $5(3,4 \%)$ & 7,86 & 6,95 \\
\hline Tabaco & $135(90,6 \%)$ & $12(8,1 \%)$ & $2(1,3 \%)$ & 2,89 & 5,55 \\
\hline
\end{tabular}

Na Tabela 1, averiguou-se que a maior frequência em termos de consumo de substâncias lícitas, como álcool e tabaco, estão representados por $69,1 \%$ de estudantes para o consumo de médio risco de álcool e 90,6\% de consumo de baixo risco de tabaco. Os estudantes que apresentaram baixo risco receberam informações de reforço do comportamento e aos estudantes que apresentaram médio ou alto risco e, assim, necessitariam de intervenção breve, foram orientados a procurar acompanhamento no serviço prestado pela própria universidade.

\section{Nivel referido de estresse}

No domínio 1, que se refere à Realização das Atividades Práticas, a prevalência dos estudantes com baixo a médio nível de estresse é aproximadamente de $40 \%$ $(\mathrm{m}=10,11, \mathrm{dp}=2,69)$. Nos demais domínios, como o D2 $(\mathrm{m}=5,81 ; \mathrm{d}=2,15)$, relativo à Comunicação Profissional, esteve representado com $43 \%$ de estudantes com baixo nível de estresse. O domínio $3(\mathrm{~m}=9,54 ; \mathrm{dp}=2,79)$, Gerenciamento de Tempo, tem $60,4 \%$ de estudantes com nível baixo de estresse e apenas $4(2,68 \%)$ com nível muito 
alto. O domínio $4(\mathrm{~m}=7,13 ; \mathrm{dp}=3,42)$, Ambiente, apresenta $51 \%$ com nível baixo de estresse e $19(12,75 \%)$ com nível muito alto de estresse. O domínio $5(\mathrm{~m}=10,55 ; \mathrm{dp}=3)$, que se refere à Formação Profissional, alcançou os níveis mais alarmantes de estresse nessa pesquisa, apresentando 59
(39,60\%) com nível baixo e $41(27,52 \%)$ com nível muito alto de estresse. O domínio $6(\mathrm{~m}=8,79 ; \mathrm{dp}=2,38)$, que mede o estresse relativo às Atividades Teóricas do curso, teve $100(67,1 \%)$ com nível baixo de estresse e 7 (4,70\%) com nível muito alto.

Tabela 2 - Correlação de Spearman e variância entre o nível de estresse, consumo referido de álcool, tabaco e série do curso de enfermagem. Londrina, PR, Brasil, 2012

\begin{tabular}{|c|c|c|c|c|c|c|}
\hline & Total & D1 & D5 & D6 & Álcool & Tabaco \\
\hline \multicolumn{7}{|l|}{ Álcool } \\
\hline$r$ & 0,109 & 0,058 & $-0,033$ & 0,034 & -0 & $0,312^{*}$ \\
\hline Variância & $10,18 \%$ & $00,33 \%$ & $00,1 \%$ & $00,11 \%$ & - & $90,73 \%$ \\
\hline$p$ valor & 0,184 & 0,485 & 0,688 & 0,682 & - & 0,000 \\
\hline \multicolumn{7}{|l|}{ Tabaco } \\
\hline$r$ & $-0,168^{\dagger}$ & $-0,209^{\dagger}$ & $-0,128$ & $-0,122$ & $0,312^{*}$ & 1,0 \\
\hline Variância & $20,82 \%$ & $40,36 \%$ & $10,63 \%$ & $10,48 \%$ & $90,73 \%$ & - \\
\hline $\mathrm{p}$ valor & 0,040 & 0,011 & 0,119 & 0,140 & 0,000 & _ \\
\hline \multicolumn{7}{|l|}{ Série } \\
\hline$r$ & 0,144 & 0,123 & $0,253^{*}$ & $-0,271^{*}$ & $-0,061$ & 0,036 \\
\hline Variância & $20,07 \%$ & $10,51 \%$ & $60,4 \%$ & $70,34 \%$ & $00,37 \%$ & $00,12 \%$ \\
\hline $\mathrm{p}$ valor & 0,081 & 0,134 & 0,002 & 0,001 & 00,458 & 0,664 \\
\hline
\end{tabular}

†A correlação é significativa no nível 0,05 (bilatel)

\section{Discussão}

\section{Estresse e formação acadêmica}

Conforme resultados da análise estatística pelos testes de Kruskal-Wallis e Jonckheere-Terpstra, foi encontrada significância estatística quando se compararam as quatro séries do curso e os seis domínios de estresse, em dois domínios: o de Formação Profissional (D5) e o de Atividade Teórica (D6). Isso significa que há diferenças de percepção e fonte geradora do estresse para cada série do curso de enfermagem estudado $(p<0,05)$. Identificou-se que há uma tendência linear positiva no domínio Formação Profissional (D5) e negativa no domínio Atividade Teórica (D6), ou seja, quanto à formação profissional, quão mais avançada a série em que o aluno se encontra maior o nível de estresse relacionado a esse aspecto. Isso significa que a preocupação com o conhecimento adquirido e o que isso irá impactar em sua futura vida profissional geram estresse cada vez maior à medida que avançam no curso $(\mathrm{JT}=3,041, \mathrm{p}<0,05)$

Com relação à atividade teórica, quanto mais avançada a série em que o aluno se encontra menor o nível de estresse, ou seja, o grau de dificuldade sentido pelos estudantes com o conteúdo programático, atividades desenvolvidas e a metodologia de ensino diminuem, sendo percebido como menor fonte estressora conforme o progredir do aluno no curso ( $\mathrm{JT}=-3,321, \mathrm{p}<0,05)$. Também, ao se analisar a Tabela 2, se nota que o domínio D5 possui uma correlação baixa com a série e o domínio D6, uma correlação baixa e negativa também com a série, também corroborando a hipótese de que no decorrer do curso o aluno apresente mais estresse pela sua formação profissional e menos estresse em relação às atividades teóricas que exerce $(r=0,253 ;-0,271, \mathrm{p}<0,01)$.
Conforme a Tabela 3, ao se verificar a variância de $6,4 \%$ em D5 e 7,34\% em D6, pode-se inferir que tais porcentagens explicam a variabilidade dos domínios em relação às séries, o que significa que 93,6\% e 92,66\% da variabilidade não se explicam por esses dois domínios de estresse mensurados pelo instrumento AEEE. Em outras palavras, o progredir do aluno, ao longo das séries do curso, é responsável por $6,4 \%$ do estresse em relação à sua preocupação sobre o conhecimento adquirido e ao impacto que esse exerce sobre sua futura vida profissional e 7,34\% do estresse em relação ao seu grau de dificuldade em lidar com o conteúdo programático, as atividades desenvolvidas e a metodologia de ensino adotada. Dessa forma, sugerese a existência de outras fontes geradoras de estresse associadas, que não as mensuradas neste estudo e neste instrumento (AEEE).

Em relação a esse achado citado acima, os seguintes estudos listam os motivos de estresse nos alunos de enfermagem: o medo em relação a dificuldades de desempenho profissional ${ }^{(14-15)}$, o receio da inserção no trabalho mercado ${ }^{(6,16)}$, a precária capacidade afetiva e social, e conflitos de relacionamento pessoais como a segunda causa, sendo apenas ultrapassada pelo medo do desemprego após o término do curso ${ }^{(6,15)}$. Consequentemente, um sentimento de fragilidade em relação ao seu futuro profissional foi bastante reforçado nessa fase, possivelmente por um sentimento de incapacidade ou por falta de garantias após o término do $\operatorname{curso}^{(6)}$.

\section{Estresse e consumo de tabaco e álcool}

Conforme os dados constantes da Tabela 1, pode-se notar que a prevalência dos estudantes que necessitariam de intervenção breve para cessar o uso de tabaco foi de $8,1 \%$ e $3,4 \%$ para provável dependência do tabaco. 
Em estudo ${ }^{(4)}$ realizado na mesma instituição em que se realizou a presente pesquisa, identificou-se correlação fraca entre o progredir do curso e o consumo de tabaco, sugerindo que os alunos referem consumir mais tabaco, conforme progridem nas séries. Mas, no entanto, segundo os dados constantes na Tabela 2, embora haja relação significativa entre o consumo de tabaco e o estresse no domínio D1, tal correlação encontrada foi baixa e negativa. Isso significa que, à medida que o aluno adquire o conhecimento instrumental para cuidar do paciente, progressivamente no curso de enfermagem, isso gera menos estresse, fazendo com que consuma menos tabaco, embora a intensidade dessa relação seja baixa $(r h o=-0,168$, $-0,209 ; \mathrm{p}<0,05)$, provavelmente, porque a prevalência do consumo frequente de tabaco também não foi acentuada.

Analisando-se a variância, segundo a Tabela 2, notase que apenas $4,36 \%$ do consumo referido de tabaco é explicado pelo estresse relacionado à aquisição de conhecimento instrumental progressivo para cuidar do paciente. Dessa forma, considera-se que a existência de outros fatores de enfrentamento ao estresse está associada ao consumo de tabaco, de forma majoritária.

Percebe-se, então, que o aumento progressivo do consumo de tabaco ${ }^{(4)}$ parece não possuir relação positiva com o nível de estresse no estudante de enfermagem, conforme havia sido hipotetizado em estudo anterior, mas parece confluir para outro estudo, feito na mesma instituição, em $2009^{(5)}$, mostrando que a percepção de estresse no aluno é alta, embora as fontes de estresse pareçam ser diferentes de outras populações de estudantes universitários de enfermagem $^{(6,16)}$, sugerindo que o estresse, embora existente no estudante de enfermagem, possui causas-fontes geradoras diferentes, principalmente as de base socioeconômica ${ }^{(10)}$.

De acordo com os dados contidos na Tabela 1, nota-se que a prevalência dos consumidores de baixo risco/abstêmios foi de $27,5 \%$, os bebedores de risco de $69,1 \%$ e os estudantes com provável dependência alcoólica foi de 3,4\%. Diversos estudos apontam tal droga como a substância psicoativa mais consumida pelos universitários $^{(1-2)}$ e também os de enfermagem ${ }^{(3-4)}$. Se comparado o consumo referido de álcool e tabaco, nota-se que há grande diferença, como também já diagnosticado em outros estudos ${ }^{(3-4,17)}$. É provável que tal diferença no consumo dessas duas drogas seja resultado da política de combate ao uso do tabaco, pela Lei Estadual n'16.239, a qual proíbe o uso de cigarros, cigarrilhas, charutos, cachimbos ou outros produtos que produzam fumaça, derivados ou não do tabaco, em ambientes de trabalho, estudo, cultura, lazer, esporte, entretenimento e em áreas comuns de condomínios ${ }^{(18)}$.

O álcool surge como substância de maior prevalência em relação ao tabaco, assim como foi visto em estudos anteriores $^{(1-4,17)}$. Um estudo ${ }^{(4)}$, realizado em 2010 na mesma instituição, lista o álcool, o tabaco e a maconha, em ordem decrescente, como as mais consumidas pelos alunos de enfermagem. O uso do tabaco em relação ao álcool é pequeno, porém, há tendência de o álcool se manter durante todos os anos e sob todas as situações de estresse ou não, na vida do aluno, o que mostra a necessidade de as universidades investirem em estratégias que afastem os seus alunos do abuso dessas substâncias.

A entrada na universidade se torna uma fase de adaptação ao cotidiano do ensino superior e metodologia de ensino, e tem a tendência de diminuir conforme o progredir das séries. Sabe-se que todo processo em que o sujeito é exposto a novas situações e com necessidade de desenvolver ou adquirir habilidades implica submetê-lo a vivência de maior ou menor intensidade de estresse.

O caráter ameaçador ou desafiador do processo ensinoaprendizagem é particularmente importante aos estudantes de enfermagem, em especial, quando o erro nesse processo implica prejuízo direto ao paciente ${ }^{(14-15)}$. No domínio 6, que avalia o estresse em relação à Atividade Teórica, nota-se que o grau de dificuldade sentido pelo estudante em relação ao currículo em si diminui no decorrer do curso, sugerindo, então, que o estudante da instituição analisada possui maior segurança ou autoconfiança, diferente de outros estudos ${ }^{(6,16)}$, nos quais os alunos evidenciaram muita preocupação e estresse com o último ano do curso, pela sobrecarga de atividades e, nessa mesma instituição analisada, o estresse pela sua inserção no mercado de trabalho ${ }^{(5)}$.

O acompanhamento dos estudantes de enfermagem nas atividades teóricas ou em práticas clínicas permite considerar as dificuldades vivenciadas pelos alunos. Inúmeros motivos podem ser destacados como fatores de estresse. Na etapa de ingresso na universidade, os alunos se deparam com um novo ambiente, diferente e distante de seu contexto de vida. A necessidade de adequação às novas exigências e obrigações escolares, as responsabilidades sociais e ocupacionais que compõem o processo de aprendizagem, a necessidade de melhor organização das tarefas diárias, o convívio com outros colegas e os desafios frequentes quanto às opções profissionais e pessoais contribuem para $\mathrm{o}$ aparecimento de situações de ansiedade e estresse ${ }^{(15)}$.

Um estudo preliminar para a construção do instrumento de mensuração do estresse no estudante de enfermagem $^{(19)}$ e outro também, realizados com alunos na última série do curso ${ }^{(6,16)}$ sugerem que os estudantes que se encontram na fase final do curso de graduação vivenciam ocasiões de medo quanto ao futuro não mais como aluno, mas como profissional, indicando amadurecimento esperado, para esse momento.

\section{Conclusões}

Diversos estudos apontaram o uso cada vez maior e mais precoce de substâncias psicoativas, principalmente o álcool e o tabaco, por parte dos estudantes universitários brasileiros. Culturalmente, o álcool e o tabaco são vistos pela sociedade como fatores de enfrentamento ao estresse, sendo ligados ao prazer, alívio e status. Percebeu-se, também, que o consumo de tabaco esteve associado ao estresse acadêmico, mas de forma contrária ao que se pressupunha, significando que quanto menor foi o estresse relacionado à aquisição do conhecimento instrumental para o cuidado maior foi o consumo de tabaco.

Com a análise estatística realizada, foi possível encontrar uma relação entre o nível de estresse associado a pontos 
específicos, como a atividade prática, formação profissional e as atividades teóricas, havendo, então, uma linearidade crescente na formação profissional, significando que, quanto mais o aluno avança nas séries do curso, maior o nível de estresse com o conhecimento adquirido e seu impacto para o futuro profissional, sugerindo seu medo com sua inserção no mercado profissional, e menor com as atividades teóricas, uma vez que o aluno já está mais adaptado à metodologia de ensino, conteúdo programático e atividades desenvolvidas. Isso significa que o aluno pode desenvolver estratégias de enfrentamento a essas fontes estressoras como uso de medicamentos, por exemplo, uma vez que a variabilidade em relação ao álcool e tabaco é muito pequena.

Enquanto limitações para este estudo se destacam a incerteza quanto à classificação do fator estresse acadêmico do estudante de enfermagem como fator de risco, uma vez que há a necessidade de realização de um estudo longitudinal, bem como ampla interferência de diversas variáveis externas sobre o estresse, dificultando seu estudo individualizado. Não se encontram estudos nacionais com o tema de estresse com análise estatística, o que dificulta a comparação com outros locais e populações.

O presente estudo, no entanto, sem dúvida, trouxe avanços significativos para o conhecimento sobre a interface entre o processo de formação do enfermeiro, o estresse associado a esse processo e o uso de substâncias psicoativas, uma vez que, através de instrumento específico para o estudante de enfermagem, foram identificadas as áreas de estresse mais prevalentes e sua associação com as séries do curso, bem como a baixa associação entre o consumo referido de álcool e tabaco e o estresse mensurado pelo instrumento AEEE, o que, até então, não havia sido feito em outro estudo, além de ser o primeiro estudo com a aplicação desse instrumento até o momento.

\section{Referências}

1. Secretaria Nacional de Políticas sobre Drogas - SENAD (BR). Gabinete de Segurança Institucional. I Levantamento Nacional sobre o uso de álcool, tabaco e outras drogas entre universitários das 27 capitais brasileiras. Brasília (DF): SENAD; 2010.

2. Pedrosa AA da Silva, Camacho LAB, Passos SRL, Oliveira R de VC de. Consumo de álcool entre estudantes universitários. Cad Saúde Pública. [Internet]. 2011 Ago [acesso 4 out 2012]; 27(8): 1611-21. Disponível em: http:// dx.doi.org/10.1590/S0102-311X2011000800016.

3. Mardegan PS, Souza RS de, Buaiz V, Siqueira MM de. Psychoactive substance use between students of the nursing. J Bras Psiquiatr. [Internet]. 2007 [acesso 13 jan 2012];56(4):260-6. Disponível em: http://dx.doi. org/10.1590/S0047-20852007000400004

4. Soares MH, Luís MAV, Corradi-Webster CM, Martins JT, Hirata AGP. Psychological concept of optimism and drug use among nursing students. Acta Paul Enferm. [Internet]. 2011[acesso 13 jan 2012];24(3):393-9. Disponível em: http://dx.doi.org/10.1590/S0103-21002011000300014

5. Barboza MC, Soares MH. Analysis of stress-causing factors in final undergraduate year of the nursing course. Acta Sci Health Sci. 2012;34(2):177-83.
6. Silva VL dos $\mathrm{S}$, Chiquito $\mathrm{N}$ do $\mathrm{C}$, Andrade RAP de O, Brito M de FP, Camelo SHH. Stress factors in the final year of undergraduate nursing: students' perceptions. Rev Enferm UERJ. 2011;19(1):121-6.

7. Seyle H. Stress: a tensão da vida. 2.ed. São Paulo: Ibrasa; 1965. 380p.

8. Lazarus RS, Folkman S. Stress appraisal and coping. New York: Springer; 1984.

9. Costa ALS, Polak C. Construction and validation of an instrument for the assessment of stress among nursing students. Rev Esc Enferm USP. 2009;43(spe):1017-26.

10. Benavente SBT, Costa ALS. Physiological and emotional responses to stress in nursing students: an integrative review of scientific literature. Acta Paul Enferm. [Internet]. 2011[acesso 13 jan 2012];24(4):5716. Disponível em: http://dx.doi.org/10.1590/S010321002011000400019

11. Henrique IFS, De Micheli D, Lacerda RB de, Lacerda LA de, Formigoni MLO de S. Validação da versão brasileira do teste de triagem do envolvimento com álcool, cigarro e outras substâncias (ASSIST). Rev Assoc Med Bras. 2004;50(2):199-206.

12. WHO ASSIST Working Group. The Alcohol, Smoking and Substance Involvement Screening Test (ASSIST): development, reliability and feasibility. Addiction. [Internet]. 2002[acesso 13 jan 2012];97(9):118394. Disponível em: http://onlinelibrary.wiley.com/ doi/10.1046/j.1360-0443.2002.00185.x/full

13. Bisquerra R, Sarriera JC, Martínez F. Introdução a estatística. Porto Alegre: Artmed; 2004. 256 p.

14. Cerchiari EAN, Caetano DFO. Prevalência de transtornos mentais menores em estudantes universitários. Estud Psicol. 2005;10(3):413-20.

15. Jorge MSB. Situações vivenciadas pelos alunos de enfermagem, durante do curso, no contexto universitário: apontadas como norteadoras de crises. Rev Esc Enferm USP. 1996;30(1):138-48.

16. Soares MH, Bueno SMV. A percepção do aluno do quarto ano de Enfermagem em relação ao seu ingresso no Mercado de Trabalho. Nursing. 2006;101(9):1064-9.

17. Botti NCL, Lima AFD, Simões WMB. Uso de substâncias psicoativas entre acadêmicos de enfermagem da Universidade Católica de Minas Gerais. SMAD, Rev. Eletrônica Saúde Mental Álcool Drog. (Ed. port.) [Internet]. 2010[acesso 08 jan 2011];6(1):1-16. Disponível em: http://pepsic.bvsalud.org/pdf/smad/v6n1/13.pdf

18. Governo do Estado do Paraná (BR). Diário Oficial do Estado. Lei 16239 - 29 de Setembro de 2009. Estabelece normas de proteção à saúde e de responsabilidade por

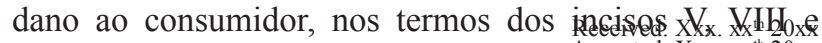

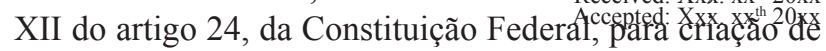
ambientes de uso coletivo livres de produtos fumígenos, conforme especifica e adota outras providências. Diário Oficial do Estado. 2009;(8066):3.

19. Costa ALS. Stress in nursing students: construction of determining factors. Rev Min Enferm. 2007;11(4):414-9. 